\title{
A Theoretical Framework for Modeling Asymmetric, Nonpositive Definite and Nonuniform Distance Functions on $\mathbf{R}^{\mathrm{n}}$
}

\author{
H. Sánchez-Larios* ${ }^{* 1}$, S. Guillén-Burguete ${ }^{2}$ \\ 1,2 Instituto de Ingeniería, Universidad Nacional Autónoma de México \\ Edificio 12 del Instituto de Ingeniería, Circuito Exterior, \\ Ciudad Universitaria, 04360 México, D. F \\ *herica.sanchez@ciencias.unam.mx
}

\begin{abstract}
In this paper, we give theoretical foundations for modeling distance functions on the usual Euclidean space $R^{n}$, where distance may refer to physical kilometers, liters of fuel consumed, time spent in traveling, or transportation cost. In our approach, a distance function $d$ is derived from a function $F_{0}$ called the fundamental function of d. Our distance functions, unlike metrics, can be asymmetric and non-positive definite, and unlike the $L p$ norms, they can be nonuniform. We illustrate our theoretical framework by modeling an asymmetric and non-uniform distance function on $R^{2}$ which can take negative values.
\end{abstract}

Keywords: Distance function, isotropy, non-uniformity, asymmetry, weighted Lp-norm

\section{RESUMEN}

En este artículo se dan bases teóricas para modelar funciones distancia sobre el espacio euclidiano usual $R^{n}$, donde distancia se puede referir a kilómetros, litros de combustible consumidos, tiempo de recorrido, o costo de transporte. En nuestro enfoque, una función distancia $d$ se obtiene a partir de una función $F_{0}$ llamada la función fundamental de d. Estas funciones distancia, a diferencia de las métricas, pueden ser asimétricas y no positivas definidas, y a diferencia de las normas $L p$, pueden ser no uniformes. Se ilustra el marco teórico propuesto a través del modelado de una función distancia asimétrica y no uniforme sobre $R^{2}$ la cual puede tomar valores negativos.

\section{Introduction}

Distance functions are involved in a wide variety of applications such as continuous location problems, distribution and transportation planning, geographical information systems, and robotics; see $[1,2]$ and references therein for a more detailed description of applications of distance functions. In most of the applications, the distance functions have been modeled by way of weighted sum of $L p$ norms (see, e.g., [3,4,5,6]). By definition, norms are symmetric, non-negative and uniform and hence the derived distance functions also have these properties.

In several real situations, symmetry, non-negativity and uniformity are violated. Real-world distances are usually more complicated than the distances determined only by the coordinates of points; in the former there are obstacles (such as rush-hour traffic, rivers, lakes or mountains) and favorable conditions (e.g., shortcuts, winds, flows, and slopes) which increase or decrease the distances, respectively. Therefore, if we want a really close description of real world distances, much more complex distance notions have to be considered.

From a theoretical framework proposed by Sánchez-Larios H and Guillén-Burguete S [7] on distance functions defined on manifolds, in this paper we develop theoretical foundations for modeling asymmetric, non-positive definite and non-uniform distance functions on $R^{n}$ (the space on which many engineering problems involving distances are formulated). In our approach, a distance function $d$ is defined in terms of a function $F_{0}$ (called the fundamental function of $d$ ) as follows: $d(\mathbf{a}, \mathbf{b})$ is the $F_{0}$-length, $\int F_{0}(\mathbf{x}(s), \dot{\mathbf{x}}(s)) d s$, of the "shortest path" connecting a to b. A distance function obtained in this way satisfies the triangle inequality and the identity property $(d(\mathbf{a}, \mathbf{a})=0)$; a distance function satisfying these two properties is 
called a premetric. Here, distance may refer to physical kilometers, liters of fuel consumed, time spent in traveling, or transportation cost.

The organization of the paper is as follows. In Section 2 , we give some concepts (such as distance function, arc length associated to a distance function, geodesic arc, and arc induced by a distance function) required to prove some theorems useful for modeling distance functions on $R^{n}$.

Some properties of a distance function (such as uniformity and isotropy) are applicable only when the manifold on which the distance function is defined is $R^{n}$. In Section 3, we show that any of these properties of a distance function is implied by the corresponding property of its respective fundamental function, e.g., we show that if a fundamental function is uniform (resp. isotropic) then the corresponding distance function remains invariant under translation (resp. invariant under rotation). In practice, a distance function can be obtained by summing several simple distance functions; from this, we prove that if an arc is a geodesic arc of several premetrics on $R^{n}$, then this arc is a geodesic arc of the sum of these premetrics.

In Section 4, we first describe a fundamental function $F_{0}$ and its premetric $d$ as formulated by [7]. Function $F_{0}$ is obtained from a simple physical model consisting of an object sliding along a path on a smooth surface $f$ embedded in the Euclidean space $R^{3} . F_{0}$ is determined by the surface $f$. Next, considering that a half-sphere is one of the simplest nonplanar surfaces, in this section attention is given to the problem of modeling a premetric on this surface, as a guide to generalization of the findings to more complex surfaces. Then, we develop the fundamental function $F_{0}$ for this surface and obtain the corresponding premetric which is asymmetric, nonuniform, and can take negative values.

\section{Basic concepts}

In this section, we give basic concepts, such as distance function, one-sided directional derivative of a distance function, arc length associated to a distance function, arc induced by a distance function, and geodesic arc. Also, we show some theorems needed for modeling distance functions.
The following properties $\mathrm{PX}, \mathrm{X}=1, \ldots, 9$ allow us to characterize binary functions. A binary function $d$ satisfies the property $\mathrm{PX}$ if for all $\mathbf{a}, \mathbf{b}, \mathbf{c}, \mathbf{u}, \mathbf{v} \in R^{n}$ :

P1. $d(\mathbf{a}, \mathbf{b}) \leq d(\mathbf{a}, \mathbf{c})+d(\mathbf{c}, \mathbf{b}) \quad$ (Triangle inequality)

P2. $d(\mathbf{a}, \mathbf{a})=0$

(Identity)

P3. $d(\mathbf{a}, \mathbf{b}) \geq 0$

(Non-negativity)

P4. $d(\mathbf{a}, \mathbf{b})=d(\mathbf{b}, \mathbf{a})$

(Symmetry)

P5. $d(\mathbf{a}, \mathbf{b})=0 \Rightarrow \mathbf{a}=\mathbf{b}$

P6. $d(\mathbf{a}+\mathbf{c}, \mathbf{b}+\mathbf{c})=d(\mathbf{a}, \mathbf{b})$

(Definiteness)

P7. $d(\mathbf{a}, \mathbf{b})=-d(\mathbf{b}, \mathbf{a})$

(Uniformity)

P8. $d(\lambda \mathbf{a}, \lambda \mathbf{b})=\lambda d(\mathbf{a}, \mathbf{b})$

for all $\lambda \in R$

(Antisymmetry)

(Positive homogeneity)

P9. $d(\mathbf{a}, \mathbf{a}+\lambda \mathbf{v})=d(\mathbf{a}, \mathbf{a}+\lambda \mathbf{u})$ for all $\lambda>0$ small

enough (Isotropy)

According to [7], a distance function $d$ on $R^{n}$ is a binary function $d: R^{n} \times R^{n} \rightarrow R$ satisfying the identity property (P2), and a premetric is a distance function $d$ on $R^{n}$ satisfying the triangle inequality (P1).

The following theorem can be proved directly. It will allow us to construct distance functions from positive linear combinations of distance functions.

Theorem 1 (Properties PX are closed under positive linear combinations). Let $d_{1}, \ldots, d_{m}$ be $m$ distance functions on $R^{n}$ satisfying the property $\mathrm{PX}$ (X $=1, \ldots, 9)$, and let $k_{1}, \ldots, k_{m}$ be $m$ non-negative real numbers. Then the distance function on $R^{n}$ defined by $d=k_{1} d_{1}+\ldots+k_{m} d_{m}$ satisfies the same property PX.

A path on $R^{n}$ from $\mathbf{a} \in R^{n}$ to $\mathrm{b} \in R^{n}$ is a continuous function $\mathbf{x}:[a, b] \rightarrow R^{n}$ where $\mathbf{x}(a)=\mathbf{a}$ and $\mathbf{x}(b)=\mathbf{b}$, and $a<b$ are real numbers. The directed image $C(\mathbf{a}, \mathbf{b}) \subseteq R^{n}$ of a path on $R^{n}$ from $\mathbf{a} \in R^{n}$ to $\mathbf{b} \in R^{n}, \mathbf{x}$ : $[a, b] \rightarrow R^{n}$, is called a directed arc on $R^{n}$ connecting $\mathbf{a}$ and $\mathbf{b}$. A parametrization of an arc $C(\mathbf{a}, \mathbf{b})$ is a path $\mathbf{x}:[a, b] \rightarrow R^{n}$ whose image is $C(\mathbf{a}, \mathbf{b})$. A parametrization of an arc is smooth if the derivative of $\mathbf{x}:[a, b] \rightarrow R^{n}$ is both continuous and not equal to zero for any $t \in[a, b]$. An arc is said to be a smooth arc if it is possible to find a smooth parametrization of $C(\mathbf{a}, \mathbf{b})$. Throughout this paper, an arc will mean a smooth directed arc; the set of all the smooth arcs on $R^{n}$ is denoted by $\Omega$. By simplicity, the set of all the smooth arcs from $\mathbf{a}$ to $\mathbf{b}$ and the set of their smooth parametrizations are denoted by $\Omega_{[a, b]}$. 
Let $d: R^{n} \times R^{n} \rightarrow R$ be a distance function on $R^{n}$. The one-sided directional derivative $F: R^{n} \times$ $R^{n} \backslash\{0\} \rightarrow R$ of the distance function $d$ at $\mathbf{x} \in R^{n}$ in the direction $\mathbf{v} \in R^{n} \backslash\{\boldsymbol{0}\}$ emanating from $\mathbf{x}$ is defined to be the limit

$$
F(\mathbf{x}, \mathbf{v})=\lim _{h \rightarrow 0^{+}} \frac{d(\mathbf{x}, \mathbf{x}+h \mathbf{v})-d(\mathbf{x}, \mathbf{x})}{h}
$$

if it exists. By the identity property of $d$, (1) becomes

$$
F(\mathbf{x}, \mathbf{v})=\lim _{h \rightarrow 0^{+}} \frac{d(\mathbf{x}, \mathbf{x}+h \mathbf{v})}{h} .
$$

The function $F: R^{n} \times R^{n} \backslash\{0\} \rightarrow R$ given by (2) evaluated at the point $\mathbf{x}$ in the direction $\mathbf{v}$ will be denoted by $F(\mathbf{x}, \mathbf{v})$, and the function $F$ along a path $\mathbf{x}:[a, b] \rightarrow R^{n}$ will be denoted by $F(\mathbf{x}(s), \dot{\mathbf{x}}(s))$ and it is given by

$$
\begin{aligned}
F(\mathbf{x}(s), \dot{\mathbf{x}}(s)) & =\lim _{h \rightarrow 0^{+}} \frac{d(\mathbf{x}(s), \mathbf{x}(s)+h \dot{\mathbf{x}}(s))}{h} \\
& =\lim _{h \rightarrow 0^{+}} \frac{d(\mathbf{x}(s), \mathbf{x}(s+h))}{h},
\end{aligned}
$$

where $\dot{\mathbf{x}}(s)$ is the derivative of $\mathbf{x}(\cdot)$ at $s$.

One can interpret the value of $F(\mathbf{x}, \mathbf{x}+d \mathbf{x})$ as the infinitesimal length of a line element going from the point $\mathbf{x} \in R^{n}$ to another point $\mathbf{x}+d \mathbf{x}$ close to $\mathbf{x}$ on the direction $d \mathbf{x} \in R^{n}$ emanating from $\mathbf{x}$. Hence, the length of an arc is determined by integrating $F$. Then we have the following theorem.

Theorem 2 (Length of an arc with respect to a distance function $d$ ). The arc length with respect to a distance function $d: R^{n} \times R^{n} \rightarrow R$ is given by

$$
l(C(\mathbf{a}, \mathbf{b}))=\int_{a}^{b} F(\mathbf{x}(s), \dot{\mathbf{x}}(s)) d s, \quad \text { for all } C(\mathbf{a}, \mathbf{b}) \in \Omega
$$

if and only if $F: R^{n} \times R^{n} \backslash\{\boldsymbol{0}\} \rightarrow R$ is the one-sided directional derivative of $d$, and it is continuous where $\mathbf{x}:[a, b] \rightarrow R^{n}$ is a smooth parametrization of the $\operatorname{arc} C(\mathbf{a}, \mathbf{b})$.
There are many practical cases in which the foregoing integral arises: (a) Certain physical examples where $\mathbf{x}(s)$ stands for position and $\dot{\mathbf{x}}(s)$ for velocity; in these cases, $F_{0}$ would have the meaning of speed, $s$ would play the role of time, and then the above integral measures distance traveled; (b) Optics problems where in an anisotropic medium the speed of light depends on its direction of travel (see, e.g., [8]). In this context, the speed of an object, in general, depends on its position and on its direction of travel; (c) Facility location problems formulated with a geometrical approach, see [9].

We call the length of the $\operatorname{arc} C(\mathbf{a}, \mathbf{b})$ with respect to a distance function $d$ the $d$-length of $C(\mathbf{a}, \mathbf{b})$. If the $d$ length of an $\operatorname{arc} C(\mathbf{a}, \mathbf{b})$ is finite, then $C(\mathbf{a}, \mathbf{b})$ is said to be $d$-rectifiable. It is immediate that all the subarcs of any subdivision of a $d$-rectifiable arc $C(\mathbf{a}, \mathbf{b})$ are $d$ rectifiable, and that the sum of their $d$-lengths is equal to the $d$-length of $C(\mathbf{a}, \mathbf{b})$.

A function $F: R^{n} \times R^{n} \backslash\{\boldsymbol{0}\} \rightarrow R$ is convex at a point $\mathbf{x} \in R^{n}$ if $F(\mathbf{x}, \alpha \mathbf{v}+(1-\alpha) \mathbf{w}) \leq \alpha F(\mathbf{x}, \mathbf{v})+(1-\alpha) F(\mathbf{x}$, $\mathbf{w})$, for all $\mathbf{v}, \mathbf{w} \in R^{n} \backslash\{\boldsymbol{0}\}, \alpha \in[0,1]$. Considering that the function $F(\mathbf{x}, \mathbf{v})$ is positively homogeneous of degree one in $\mathbf{v}, F$ is convex at $\mathbf{x}$ if and only if $F(\mathbf{x}, \mathbf{v}$ $+\mathbf{w}) \leq F(\mathbf{x}, \mathbf{v})+F(\mathbf{x}, \mathbf{w})$, for all $\mathbf{v}, \mathbf{w} \in R^{n} \backslash\{\mathbf{0}\}$. Function $F$ is said to be a convex function if $F$ is convex at all $\mathbf{x} \in R^{n}$.

Theorem 3 (Properties of the one-sided directional derivative $F$ of a distance function $d$ ). Let $d: R^{n} \times R^{n} \rightarrow R$ be a distance function, and let $F: R^{n} \times R^{n} \backslash\{\mathbf{0}\} \rightarrow R$ be the one-sided directional derivative $F$ of $d$. Then we have:

(a) The function $F$ is positively homogeneous of degree one in $\mathbf{v}$, i.e., $F(\mathbf{x}, \alpha \mathbf{v})=\alpha F(\mathbf{x}, \mathbf{v})$ for all $\alpha>0$ and for all $\mathbf{x} \in R^{n}$ and $\mathbf{v} \in R^{n} \backslash\{\mathbf{0}\}$.

(b) For $n \geq 2 F(\mathbf{x}(s), \dot{\mathbf{x}}(s))$ does not depend explicitly on the parameter $s$ of the path.

(c) The d-length of a d-rectifiable arc does not depend on the parametric representation of the arc.

(d) If $d$ satisfies the triangle inequality, then $F$ is convex, i.e., $F(\mathbf{x}, \alpha \mathbf{v}+(1-\alpha) \mathbf{w}) \leq \alpha F(\mathbf{x}, \mathbf{v})+(1-$ $\alpha) F(\mathbf{x}, \mathbf{w})$ for all $\alpha \in[0,1]$. 
Proof:

(a) For $\alpha>0$,

$$
\begin{aligned}
F(\mathbf{x}, \alpha \mathbf{v}) & =\lim _{h \rightarrow 0^{+}} \frac{d(\mathbf{x}, \mathbf{x}+\alpha h \mathbf{v})}{h}=\alpha \lim _{h \rightarrow 0^{+}} \frac{d(\mathbf{x}, \mathbf{x}+\alpha h \mathbf{v})}{\alpha h} \\
& =\alpha \lim _{\beta \rightarrow 0^{+}} \frac{d(\mathbf{x}, \mathbf{x}+\beta \mathbf{v})}{\beta}=\alpha F(\mathbf{x}, \mathbf{v}) .
\end{aligned}
$$

(b) As one can see, the one-sided directional derivative $F$ of the distance function $d$ along the path $\mathbf{x}:[a, b] \rightarrow R^{n}$, given by (3), does not depend explicitly on $s$.

(c) The $d$-length of a $d$-rectifiable arc is given by (4). Let $s=s(t)$ be an orientation-preserving transformation of $\mathbf{x}(t)$. Therefore $d s / d t>0$ and $s(t)$ is an invertible function. The positive homogeneity of $F$ and $d s / d t>0$ imply that $F(\boldsymbol{x}, d \boldsymbol{x}(t) / d t) d t=F(\boldsymbol{x}, \dot{\boldsymbol{x}} d s / d t) d t$ $=F(\boldsymbol{x}, \dot{\boldsymbol{x}})(d s / d t) d t=F(\boldsymbol{x}, \dot{\boldsymbol{x}}) d s$. Since $F$ does not depend explicitly on the parameter $s, F(x(s), \dot{\boldsymbol{x}}(s)) d s$ is invariant under the transformation $s=s(t)$.

(d) From equation (2) and using (5), we obtain $F(\mathbf{x}$, $\alpha \mathbf{v}+(1-\alpha) \mathbf{w}) \leq \alpha F(\mathbf{x}, \mathbf{v})+(1-\alpha) F(\mathbf{x}, \mathbf{w})$ for all $\alpha \in[0,1]$.

An $\operatorname{arc} C(\mathbf{a}, \mathbf{b})$ is a geodesic arc with respect to a distance function $d$ (d-geodesic arc) if it is $d$ rectifiable and its $d$-length is at least as small as the $d$-length of any other arc going from $\mathbf{a}$ to $\mathbf{b}$. It is immediate that each subarc of a $d$-geodesic arc is a $d$-geodesic arc. A distance function $d$ is complete if for every ordered pair of points $(\mathbf{a}, \mathbf{b})$ in $R^{n}$ there exists at least one smooth $d$-geodesic arc connecting $\mathbf{a}$ and $\mathbf{b}$.

It is worth to notice that the left-hand side of the triangle inequality $d(\mathbf{a}, \mathbf{c})+d(\mathbf{c}, \mathbf{b})-d(\mathbf{a}, \mathbf{b}) \geq 0$ reaches its minimum value when it is equal to zero. Thus, the following definition, which will be used to characterize the geodesic arcs, makes sense.

We define an arc induced by a distance function $d(d-$ induced arc) as an arc $C(\mathbf{a}, \mathbf{b})$ holding, for a parametric representation of the $\operatorname{arc} C(\mathbf{a}, \mathbf{b}), \mathbf{x}:[a, b] \rightarrow$ $R^{n}$, with $\mathbf{x}(a)=\mathbf{a}$ and $\mathbf{x}(b)=\mathbf{b}$, the triangle equality:

$$
d(\mathbf{x}(s), \mathbf{b})=d(\mathbf{x}(s), \mathbf{x}(t))+d(\mathbf{x}(t), \mathbf{b}), \quad \text { for all } s \in[a,
$$
$b]$ and for all $t \in[s, b]$.

It can be proved directly that expression (6) is equivalent to any of the following two conditions:

$$
\begin{gathered}
d(\mathbf{x}(s), \mathbf{x}(z))=d(\mathbf{x}(s), \mathbf{x}(t))+d(\mathbf{x}(t), \mathbf{x}(z)), \\
\text { where } a \leq s \leq t \leq z \leq b, \\
d(\mathbf{x}(s), \mathbf{x}(r))-d(\mathbf{x}(s), \mathbf{x}(t))=d(\mathbf{x}(t), \mathbf{x}(z))-d(\mathbf{x}(r), \\
\mathbf{x}(z)), \text { where } a \leq s \leq t \leq r \leq z \leq b .
\end{gathered}
$$

Condition (7) expresses the triangle equality for any three ordered points on $C(\mathbf{a}, \mathbf{b})$.

In the following theorem, we prove that if a distance function is complete and satisfies the triangle inequality, then every $d$-geodesic arc is a $d$-induced arc.

\section{A premetric derived from a given function}

The proposed approach to model a premetric from a given function is based on the fact that any complete distance function satisfying the triangle inequality (P1) and the identity property (P2) can be represented by a function $F_{0}(\mathbf{x}, \mathbf{v})$ depending on the position $\mathbf{x} \in R^{n}$ and on a direction $\mathbf{v} \in R^{n}$ attached at $\mathbf{x}$ :

$d(\mathbf{a}, \mathbf{b})=\min _{\mathbf{x} \in \Omega_{[a, b]}} \int_{\mathbf{a}}^{\mathbf{b}} F_{0}(\mathbf{x}(s), \dot{\mathbf{x}}(s)) d s$, for all $\mathbf{a}, \mathbf{b} \in R^{n}$

Function $F_{0}$ is called the fundamental function of $d$, which is not necessarily the one-sided directional derivative of $d$. It can be proved that $F_{0}$ is the onesided directional derivative $F$ of $d$ if, and only if, $F_{0}$ is convex.

Notice that a premetric obtained from a fundamental function $F_{0}$ through (8) may not satisfy the conditions of nonnegativity (P3), symmetry (P4), definiteness (P5), and uniformity (P6). 
Theorem 4 (A d-geodesic arc is a d-induced arc if $d$ is a complete premetric). Let $d: R^{n} \times R^{n} \rightarrow R$ be a complete premetric. If $C(\mathbf{a}, \mathbf{b})$ is a geodesic arc of the premetric $d$, then $C(\mathbf{a}, \mathbf{b})$ is a $d$-induced arc.

\section{Proof:}

Let $\mathbf{x}:[a, b] \rightarrow R^{n}$ be a smooth parametric representation of the geodesic arc $C(\mathbf{a}, \mathbf{b})$, and let $s$, $t$ and $z$ be real numbers such that $a \leq s \leq t \leq z \leq b$. The subarcs $C(\mathbf{x}(s), \mathbf{x}(t))$ and $C(\mathbf{x}(t), \mathbf{x}(z))$ are also geodesic arcs, and the sum of their lengths is equal to the length of the arc $C(\mathbf{x}(s), \mathbf{x}(z))$, i.e., I $(C(\mathbf{x}(s)$, $\mathbf{x}(z))=I(C(\mathbf{x}(s), \mathbf{x}(t))+I(C(\mathbf{x}(t), \mathbf{x}(z))$. Due to $C(\mathbf{x}(s)$, $\mathbf{x}(t))$ and $C(\mathbf{x}(t), \mathbf{x}(z))$ are geodesic arcs, i.e., $C(\mathbf{x}(s)$, $\mathbf{x}(t))$ and $C(\mathbf{x}(t), \mathbf{x}(z))$ are arcs of minimum $d$-length, then by (8) and due to that $d$ is a complete premetric, $d(\mathbf{x}(s), \mathbf{x}(t))=I(C(\mathbf{x}(s), \mathbf{x}(t)))$ and $d(\mathbf{x}(t)$, $\mathbf{x}(z))=I(C(\mathbf{x}(t), \mathbf{x}(z)))$. Hence, condition (7) is satisfied, and therefore $C(\mathbf{a}, \mathbf{b})$ is a $d$-induced arc.

The geodesic arcs solving (8) can be found by solving the Euler-Lagrange equations:

$$
\frac{\partial F_{0}}{\partial x_{i}}-\frac{d}{d s} \frac{\partial F_{0}}{\partial \dot{x}_{i}}=0, \quad i=1,2, \ldots, n
$$

The distance functions involved in many applications are defined on the two-dimensional Euclidean space $R^{2}$. For $n=2$ the coordinates of each point can be denoted by $(x, y)$; in these terms, equations (4), (8) and (9) induce to the following Euler-Lagrange equations:

$-\frac{\partial F_{0}}{\partial x}+\left(\frac{\partial^{2} F_{0}}{\partial \dot{x} \partial x}\right) \dot{x}+\left(\frac{\partial^{2} F_{0}}{\partial \dot{x} \partial y}\right) \dot{y}+\left(\frac{\partial^{2} F_{0}}{\partial \dot{x}^{2}}\right) \ddot{x}+\left(\frac{\partial^{2} F_{0}}{\partial \dot{x} \partial \dot{y}}\right) \ddot{y}=0$,

$-\frac{\partial F_{0}}{\partial y}+\left(\frac{\partial^{2} F_{0}}{\partial \dot{y} \partial x}\right) \dot{x}+\left(\frac{\partial^{2} F_{0}}{\partial \dot{y} \partial y}\right) \dot{y}+\left(\frac{\partial^{2} F_{0}}{\partial \dot{y} \partial \dot{x}}\right) \ddot{x}+\left(\frac{\partial^{2} F_{0}}{\partial \dot{y}^{2}}\right) \ddot{y}=0$.

The $d$-length of an $\operatorname{arc} C(\mathbf{a}, \mathbf{b})$ in $R^{2}$ can be expressed in terms of the variable $y$. This is because of the positive homogeneity of degree one in $\dot{x}$ and $\dot{y}$ of $F_{0}$. Thus, the Euler-Lagrange equations (10) and (11) are reduced to

$$
-\frac{d}{d x} \frac{\partial F_{0}}{\partial y}+\left(\frac{\partial^{2} F_{0}}{\partial y^{\prime 2}}\right) y^{\prime \prime}+\left(\frac{\partial^{2} F_{0}}{\partial y^{\prime} \partial y}\right) y^{\prime}+\left(\frac{\partial^{2} F_{0}}{\partial y^{\prime} \partial x}\right)=0 .
$$

3.1 Properties of a premetric deduced from properties of its fundamental function

In practice, it is useful to know that some properties of a premetric, such as uniformity (P6), isotropy (P9), symmetry (P4), and antisymmetry (P7), can be deduced from the corresponding property of its fundamental function.

Let $d$ be a complete premetric on $R^{n}, n \geq 2$. The premetric $d$ is isotropic at $\mathbf{x} \in R^{n}$ if its fundamental function $F$ satisfies $F(\mathbf{x}, \mathbf{v} /\|\mathbf{v}\|)=F(\mathbf{x}, \mathbf{u} /\|\mathbf{u}\|)$ for all $\mathbf{u}, \mathbf{v} \in R^{n} \backslash\{\mathbf{0}\}$, i.e., $F(\mathbf{x}, \mathbf{v})=F(\mathbf{x}, \mathbf{u})$ for all $\mathbf{u}, \mathbf{v} \in R^{n}$ $\mid\{\mathbf{0}\}$ with $\|\mathbf{v}\|=\|\mathbf{u}\|$; otherwise, $d$ is anisotropic at $\mathbf{x} \in R^{n}$. Isotropy means that the fundamental function $F(\mathbf{x}, \mathbf{v})$ does not depend on the direction $\mathbf{v}$. If $d$ is isotropic at every point of its domain, we say that $d$ is isotropic on $R^{n}$, in whose case $F(\mathbf{x}, \mathbf{v})$ does not depend on $\mathbf{v}$ for all $\mathbf{x} \in R^{n}$. If $d$ is not isotropic on $R^{n}$ then it is anisotropic on $R^{n}$. A fundamental function $F$ is uniform (invariant under translation) if, and only if, $F(\mathbf{x}, \mathbf{v})=F(\mathbf{y}, \mathbf{v})$ for all $\mathbf{x}$, $\mathbf{y} \in R^{n}$ and for all $\mathbf{v} \in R^{n} \backslash\{\mathbf{0}\}$. The following theorem establishes relations between properties of a premetric $d$ and properties of its fundamental function $F$.

Theorem 5 (Relations between properties of a premetric and properties of its fundamental function). Let $d: R^{n} \times R^{n} \rightarrow R$ be a premetric and let $F: R^{n} \times R^{n} \backslash\{\boldsymbol{0}\} \rightarrow R$ the fundamental function of $d$. Then we have:

(a) Invariance under translation: If $F(\mathbf{x}, \mathbf{v})=F(\mathbf{y}, \mathbf{v})$ for all $\mathbf{x}, \mathbf{y} \in R^{n}$ and for all $\mathbf{v} \in R^{n} \backslash\{\mathbf{0}\}$, then d remains invariant under translation, i.e., $d(\mathbf{a}+\mathbf{c}, \mathbf{b}+\mathbf{c})=$ $d(\mathbf{a}, \mathbf{b})$ for all $\mathbf{a}, \mathbf{b}, \mathbf{c} \in R^{n}$.

(b) Invariance under changes of direction: If $F(\mathbf{x}, \mathbf{v})$ $=F(\mathbf{x}, \mathbf{u})$ for all $\mathbf{x} \in R^{n}$ and for all $\mathbf{u}, \mathbf{v} \in R^{n} \backslash\{\mathbf{0}\}$ with $\|\mathbf{v}\|=\|\mathbf{u}\|$, then $d$ is an isotropic premetric, i.e., $d(\mathbf{x}, \mathbf{x}+\lambda \mathbf{v})=d(\mathbf{x}, \mathbf{x}+\lambda \mathbf{u})$ for all $\mathbf{u}, \mathbf{v} \in R^{n} \backslash\{\mathbf{0}\}$ with $\|\mathbf{v}\|=\|\mathbf{u}\|$, and for all $\lambda>0$ small enough. 
(c) Invariance under inversions of direction: If $F(\mathbf{x}$, - $\mathbf{v})=F(\mathbf{x}, \mathbf{v})$ for all $\mathbf{x} \in R^{n}$ and for all $\mathbf{v} \in R^{n} \backslash\{\mathbf{0}\}$, then $d$ is a symmetric premetric, i.e., $d(\mathbf{a}, \mathbf{b})=d(\mathbf{b}, \mathbf{a})$ for all $\mathbf{a}, \mathbf{b} \in R^{n}$.

(d) If $F(\mathbf{x},-\mathbf{v})=-F(\mathbf{x}, \mathbf{v})$ for all $\mathbf{x} \in R^{n}$ and for all $\mathbf{v} \in R^{n} \backslash\{\mathbf{0}\}$, then $d$ is an antisymmetric premetric, i.e., $d(\mathbf{a}, \mathbf{b})=-d(\mathbf{b}, \mathbf{a})$ for all $\mathbf{a}, \mathbf{b} \in R^{n}$.

\section{Proof:}

(a) Let $\mathbf{x}:[a, b] \rightarrow R^{n}$ be a parametrization of the arc $C(\mathbf{a}, \mathbf{b})$.We assume that, for all $\mathbf{x}, \mathbf{y} \in R^{n}$ and for all $\mathbf{v} \in R^{n} \backslash\{\mathbf{0}\}, F(\mathbf{x}, \mathbf{v})=F(\mathbf{y}, \mathbf{v})$. Then, for all $\mathbf{c} \in R^{n}$ the arc $C(\mathbf{a}+\mathbf{c}, \mathbf{b}+\mathbf{c})$ admits the parametrization $\mathbf{y}$ : $[a, b] \rightarrow R^{n}$, where $\mathbf{y}(s)=\mathbf{x}(s)+\mathbf{c}$ implies that $\dot{\mathbf{y}}(s)=\dot{\mathbf{x}}(s)$. Then, the integrand of (4) for the arc $C(\mathbf{a}, \mathbf{b})$ is equal to the integrand for the $\operatorname{arc} C(\mathbf{a}+\mathbf{c}$, $\mathbf{b}+\mathbf{c})$, that is to say, $F(\mathbf{y}(s), \dot{\mathbf{y}}(s))=$ $F(\mathbf{x}(s)+\mathbf{c}, \dot{\mathbf{x}}(s))=F(\mathbf{x}(s), \dot{\mathbf{x}}(s))$, and therefore $I(C(\mathbf{a}, \mathbf{b}))=I(C(\mathbf{a}+\mathbf{c}, \mathbf{b}+\mathbf{c}))$. Then, by equation (8), $\int F(\mathbf{y}(s), \dot{\mathbf{y}}(s)) d s=\int F(\mathbf{x}(s)+\mathbf{c}, \dot{\mathbf{x}}(s)) d s$ implies that $\min \int F(\mathbf{y}(s), \dot{\mathbf{y}}(s)) d s=\min \int F(\mathbf{x}(s)+\mathbf{c}, \dot{\mathbf{x}}(s)) d s$, and therefore, $d(\mathbf{a}, \mathbf{b})=d(\mathbf{a}+\mathbf{c}, \mathbf{b}+\mathbf{c})$.

(b) If $F(\mathbf{x}, \mathbf{v})=F(\mathbf{x}, \mathbf{u})$ for all $\mathbf{x} \in R^{n}$ and for all $\mathbf{u}, \mathbf{v} \in R^{n}$ $\mid\{\mathbf{0}\}$ with $\|\mathbf{v}\|=\|\mathbf{u}\|$, then by using equation (2), $d(\mathbf{x}$, $\mathbf{x}+\lambda \mathbf{v})=d(\mathbf{x}, \mathbf{x}+\lambda \mathbf{u})$ for all $\mathbf{u}, \mathbf{v} \in R^{n} \mid\{\mathbf{0}\}$ with $\|\mathbf{v}\|=$ $\|\mathbf{u}\|$, and for $a \lambda>0$ small enough.

(c) Assume that $F(\mathbf{x},-\mathbf{v})=F(\mathbf{x}, \mathbf{v})$ for all $\mathbf{x} \in R^{n}$ and for all $\mathbf{v} \in R^{n} \backslash\{0\}$. Let $\mathbf{x}:[a, b] \rightarrow R^{n}$ and $\mathbf{y}:\left[a^{\prime}, b^{\prime}\right] \rightarrow$ $R^{n}$ be two parametrizations of the $\operatorname{arc} C(\mathbf{a}, \mathbf{b})$, and let $s:\left[a^{\prime}, b^{\prime}\right] \rightarrow[a, b]$ be a continuous and strictly decreasing real-valued function on $\left[a^{\prime}, b^{\prime}\right]$, with image $[a, b]$ such that $\mathbf{y}(t)=\mathbf{x}(s(t))$ where $a^{\prime} \leq t \leq b^{\prime}$. Then $\mathbf{x}$ and $\mathbf{y}$ trace out $C(\mathbf{a}, \mathbf{b})$ in opposite directions. By the chain rule, $\dot{\mathbf{y}}(t)=\dot{\mathbf{x}}(s(t)) d s / d t$, where $d s / d t$ is strictly negative, and due to the positive homogeneity of $F: F(\mathbf{y}(t), \dot{\mathbf{y}}(t)) d t=F(\mathbf{x}(s(t)), \dot{\mathbf{x}}(s(t))$ $d s / d t) d t=F(\mathbf{x}(s(t)),-\dot{\mathbf{x}}(s(t)))(-d s / d t) d t=F(\mathbf{x}(s(t))$, $-\dot{\mathbf{x}}(s(t)))(-d s)=F(\mathbf{x}(s(t)), \dot{\mathbf{x}}(s(t)))(-d s)$. Due to $d s$ and $d t$ have opposite directions, then by $(4) /(\mathbf{x})=$ $I(\mathbf{y})$, and therefore $d(\mathbf{a}, \mathbf{b})=d(\mathbf{b}, \mathbf{a})$.

(d) This is proved in the same way that part (c), but in this case, the fundamental function is $F(\mathbf{x},-\mathbf{v})=-$
$F(\mathbf{x}, \mathbf{v})$. Then, $F$ holds $F(\mathbf{y}(t), \dot{\mathbf{y}}(t)) d t=F(\mathbf{x}(s(t))$, $\dot{\mathbf{x}}(s(t)) d s / d t) d t=F(\mathbf{x}(s(t)),-\dot{\mathbf{x}}(s(t)))(-d s / d t) d t=$ $F(\mathbf{x}(s(t)),-\dot{\mathbf{x}}(s(t)))(-d s)=-F(\mathbf{x}(s(t)), \quad \dot{\mathbf{x}}(s(t)))(-$ $d s)$. Due to $d s$ and $d t$ have opposite directions, then by $(4), I(\mathbf{x})=-l(\mathbf{y})$, and therefore $d(\mathbf{a}, \mathbf{b})=-d(\mathbf{b}, \mathbf{a})$.

3.2 Geodesic arcs associated to a sum of premetrics on $R^{n}$

The following theorem and its corollary are required for modeling a premetric on $R^{n}$ from its fundamental function $F$. Recall that any sum of nonnegative premetrics on $R^{n}$ is a premetric on $R^{n}$ (Theorem 1).

Theorem 6 (Geodesic arcs of a sum of premetrics). Let $d_{1}$ and $d_{2}$ be two premetrics on $R^{n}$, and let $C(\mathbf{a}, \mathbf{b}) \subseteq R^{n}$ be a geodesic arc of each of the premetrics $d_{1}$ and $d_{2}$. Then $C(\mathbf{a}, \mathbf{b})$ is a geodesic arc of the premetric $d=d_{1}+d_{2}$.

\section{Proof}

Let $d$ be the premetric defined by $d(\mathbf{a}, \mathbf{b})=d_{1}(\mathbf{a}, \mathbf{b})$ $+d_{2}(\mathbf{a}, \mathbf{b})$ for all $\mathbf{a}, \mathbf{b} \in R^{n}$. If $C(\mathbf{a}, \mathbf{b})$ is a geodesic arc of the premetrics $d_{1}$ and $d_{2}$, then by Theorem $4, C(\mathbf{a}, \mathbf{b})$ is both a $d_{1}$-induced arc and a $d_{2}$ induced arc, i.e., $d_{1}$ and $d_{2}$ satisfy (6), then the distance function $d=d_{1}+d_{2}$ also satisfies (6); thus $C(\mathbf{a}, \mathbf{b})$ is a geodesic arc of $d$.

Any real-valued function on $R^{n}$ determines a premetric with the property that all the arcs on $R^{n}$ are geodesic arc of this premetric. Let $h: R^{n} \rightarrow R$ be a real-valued function. The binary function $d_{h}$ : $R^{n} \times R^{n} \rightarrow R$, defined by

$$
d_{h}(\mathbf{a}, \mathbf{b})=h(\mathbf{b})-h(\mathbf{a}) \quad \text { for all } \mathbf{a}, \mathbf{b} \in R^{n},
$$

satisfies the triangle equality $d_{h}(\mathbf{a}, \mathbf{b})=d_{h}(\mathbf{a}, \mathbf{c})+$ $d_{h}(\mathbf{c}, \mathbf{b})$ for all $\mathbf{a}, \mathbf{b}, \mathbf{c} \in R^{n}$, and the identity property. Therefore $d_{h}$ is a premetric which we call premetric of the real-valued function $h$. This premetric $d_{h}$ is antisymmetric and hence the sum of $d_{h}$ and any premetric $d$ is an asymmetric premetric. It can be proved directly that all the arcs going from $\mathbf{a} \in R^{n}$ to $\mathbf{b} \in R^{n}$ are arcs induced by the premetric $d_{h}$. Therefore, all the arcs going from $\mathbf{a}$ to $\mathbf{b}$ have the same length with respect to the premetric $d_{h}$ : If the 
function $h$ is differentiable, then the one-sided directional derivative of the premetric $d_{h}$ becomes

$$
\begin{aligned}
F_{h}(\mathbf{x}, \dot{\mathbf{x}}) & =\lim _{t \rightarrow 0^{+}} \frac{d_{h}(\mathbf{x}, \mathbf{x}+\dot{\mathbf{x}} t)}{t}=\lim _{t \rightarrow 0^{+}} \frac{h(\mathbf{x}+\dot{\mathbf{x}} t)-h(\mathbf{x})}{t} \\
& =(\nabla h) \cdot \dot{\mathbf{x}}, \text { for all } \mathbf{x} \in R^{n} \text { and for all } \dot{\mathbf{x}} \in R^{n},
\end{aligned}
$$

where $\nabla h$ is the gradient vector of $h$ and $\cdot$ denotes the inner product.

Corollary of Theorem 6. Let $d_{h}$ be the premetric of a real-valued function $h: R^{n} \rightarrow R$, and let $d_{F}$ be a premetric on $R^{n}$. Then, the geodesic arcs of $d_{F}$ and the geodesic arcs of the premetric $d=d_{F}+d_{h}$ are the same.

Notice that if $d_{F}$ is a symmetric premetric and $h$ is not a constant function, then the premetric $d=d_{F}+$ $d_{h}$ is an asymmetric premetric.

\section{Modeling of a premetric on $r 2$ from a fundamental function}

In order to exemplify our theoretical framework, we model a non-uniform, asymmetric and non-positive definite premetric $d$ on $R^{2}$. Our premetric is obtained from a fundamental function $F_{0}$ determined by a smooth surface $f$. Function $F_{0}$ is derived from a simple physical model consisting of an object sliding along a path on $f$. Considering that a half-sphere is one of the simplest examples of nonplanar surfaces, we model the premetric $d$ for this particular surface, as a guide to generalization of the findings to more complex surfaces.

\subsection{Modeling of a fundamental function in terms of an arbitrary surface}

In this subsection, we describe a fundamental function $F_{0}$ and its corresponding premetric $d$ as formulated by [7]. This function $F_{0}$ is determined by a smooth surface embedded in $R^{3}$ given by a function $f$ defined on the horizontal plane $R^{2} . F_{0}$ is deduced from the following simple physical model: Suppose that an object is slid on the surface $f$. Assuming that the object has no acceleration over the path, and that path turns altogether involve insignificant energy loss, then the major external forces operating on the object are gravity and friction. This model can be interpreted in terms of (8) as follows: The quantity $F_{0}(\mathbf{x}(s), \dot{\mathbf{x}}(s)) d s$ represents the energy required to slide the object on $f$ from the point $f(\mathbf{x}(s))$ on $f$ to the neighboring point $f(\mathbf{x}(s)+\dot{\mathbf{x}}(s) d s)$ on $f$, where $\dot{\mathbf{x}}(s)$ is the projection of the tangent vector to the curve on $f$ at $f(\mathbf{x}(s))$ onto the horizontal plane. The integral in (8) along a given curve $\mathbf{x}:[a, b] \rightarrow R^{2}$ on the horizontal plane joining $\mathrm{a} \in R^{2}$ to $\mathrm{b} \in R^{2}$ is the total energy needed to slide the object from $(\mathbf{a}, f(\mathbf{a}))$ to (b, $f(\mathbf{b}))$ along the corresponding curve on the surface $z=f(x, y)$. Thus the distance $d(\mathbf{a}, \mathbf{b})$ from $\mathbf{a} \in R^{2}$ to $\mathbf{b} \in R^{2}$ is the minimum energy required to slide the object from $(\mathbf{a}, f(\mathbf{a}))$ to $(\mathbf{b}, f(\mathbf{b}))$ on the surface $f$. The energy needed to slide the object from the point $(x, y)$ to another point in the $X Y$ plane at a Euclidean distance $\Delta s$ in the direction $(\dot{x}, \dot{y})$ is $F_{0}(x, y, \dot{x}, \dot{y}) \Delta s:=\Delta W$, i.e.,

$$
F_{0}(x, y, \dot{x}, \dot{y})=d W / d s
$$

Function $F_{0}$ is obtained as follows. Let $\Delta W_{g}$ and $\Delta W_{f}$ be the energies required to overcome the gravity force and the friction force, respectively. Then $\Delta W_{g}=m g \Delta f=m g[\Delta x(\partial f / \partial x)+\Delta y(\partial f / \partial y)]$ and $\Delta W_{f}=\mu m g \cos \theta\left[(\Delta x)^{2}+(\Delta y)^{2}+(\Delta x \partial f / \partial x+\Delta y \partial f\right.$ $\left.\mid \partial y)^{2}\right]^{1 / 2}$, where $m$ is the mass of the object, $\theta$ denotes the angle between the tangent plane to $f$ at $(x, y, f(x, y))$ and the horizontal plane, $g$ denotes the gravitation acceleration constant, and $\mu$ is the coefficient of sliding friction, which is supposed a constant parameter. By simplicity, it is considered that $m g=1$. Since $\cos \theta=\left[1+(\partial f / \partial x)^{2}+(\partial f / \partial y)^{2}\right]^{-}$ $1 / 2$, the total energy is $\Delta W=\Delta x(\partial f / \partial x)+\Delta y(\partial f / \partial y)+$ $\mu\left[1+(\partial f / \partial x)^{2}+(\partial f / \partial y)^{2}\right]^{-1 / 2} \cdot\left\{(\Delta x)^{2}+(\Delta y)^{2}+[\Delta x(\partial f\right.$ $\left./ \partial x)+\Delta y(\partial f / \partial y)]^{2}\right\}^{1 / 2}$. Substituting $\Delta W$ into (15), and considering that $\dot{x}^{2}+\dot{y}^{2}=1$, the fundamental function $F_{0}$ becomes

$$
\begin{aligned}
F_{0}(x, y, \dot{x}, \dot{y}) & =\dot{x}(\partial f / \partial x)+\dot{y}(\partial f / \partial y) \\
& +\mu\left[1+(\partial f / \partial x)^{2}+(\partial f / \partial y)^{2}\right]^{-1 / 2} \\
& \cdot\left\{1+[\dot{x}(\partial f / \partial x)+\dot{y}(\partial f / \partial y)]^{2}\right\}^{1 / 2} .
\end{aligned}
$$

Observe that $F_{0}$ in (16) can be seen as the sum of two expressions each corresponding to a premetric. The first expression corresponding to 
the sum of the first two terms in (16), $\dot{x}(\partial f / \partial x)+\dot{y}(\partial f / \partial y)$, is the gradient vector of $f$, and by (14) it corresponds to the premetric $d_{f}$ of the real-valued function $f$. Then, this premetric is given by $d_{f}(\mathbf{a}, \mathbf{b})=f(\mathbf{b})-f(\mathbf{a})$ for all $\mathbf{a}, \mathbf{b} \in R^{2}$, which is antisymmetric. The second expression, which corresponds to the third term in (16), $F_{\mu}(x, y, \dot{x}, \dot{y})$ $:=\mu\left[1+(\partial f / \partial x)^{2}+(\partial f / \partial y)^{2}\right]^{-1 / 2} \cdot\{1+[\dot{x}(\partial f / \partial x)+\dot{y}$ $\left.(\partial f / \partial y)]^{2}\right\}^{1 / 2}$, is continuous and positively homogeneous of degree one in $(\dot{x}, \dot{y})$. Therefore $F_{\mu}$ is well-defined, and then the corresponding premetric $d \mu$ can be derived from $F_{\mu}$ through (8).

By Corollary of Theorem 6 , the geodesic arcs of the premetric $d(\mathbf{a}, \mathbf{b})=d_{f}(\mathbf{a}, \mathbf{b})+d_{\mu}(\mathbf{a}, \mathbf{b})$ corresponding to $F_{0}$ are the same that the geodesic arcs of the premetric $d_{\mu}$. Therefore, the determination of the geodesic arcs of $d$ is reduced to the determination of the geodesic arcs of $d_{\mu}$. These geodesic arcs can be obtained by solving the Euler-Lagrange equation (12).

As one can see, for each surface $f$ in (16) there is a fundamental function $F_{0}$, and the corresponding premetric $d$ is obtained through (8). If the surface $f$ is a plane (the simplest example of a surface), the work function obtained by Hodgson et al. [10], formulated in the context of a facility location problem on an inclined plane, is a particular premetric derived from the fundamental function (16).

\subsection{Modeling of a premetric from a fundamental} function in terms of a half-sphere

In this subsection, we model a premetric on a halfsphere. We first particularize the fundamental function (16) to this surface, and then we obtain the corresponding premetric.

Suppose that in the above-described physical model, the surface on which the object slides is a half-sphere. We are going to model the corresponding premetric $d$ as the sum of two premetrics: $d=d_{F}+d_{h}$. In this case, $d_{F}$ is the premetric obtained by considering that the object is not under the action of gravity (therefore, $d_{F}$ depends only on the surface $f$ ), and $d_{h}$ is the premetric obtained by considering only the gravity (therefore $d_{h}$ is independent of the surface $f$ ).
In order to model the premetric $d_{F}$, it is considered that the object is not under the action of gravity;

therefore, $\dot{x}(\partial f / \partial x)+\dot{y}(\partial f / \partial y)=0$, and then (16) becomes:

$$
\begin{aligned}
F_{0}(x, y, \dot{x}, \dot{y})= & \mu\left[1+(\partial f / \partial x)^{2}+(\partial f / \partial y)^{2}\right]^{-1 / 2} \\
& \cdot\left\{1+[\dot{x}(\partial f / \partial x)+\dot{y}(\partial f / \partial y)]^{2}\right\}^{1 / 2}
\end{aligned}
$$

Consider a half-sphere of radius $r$ given by the function $f(x, y)=\left(r^{2}-x^{2}-y^{2}\right)^{1 / 2}$ whose domain is the open disk $D \subseteq R^{2}$ given by $x^{2}+y^{2}<r^{2}$. Substituting the function $f(x, y)=\left(r^{2}-x^{2}-y^{2}\right)^{1 / 2}$ into the fundamental function (17), and considering that $\dot{x}^{2}+\dot{y}^{2}=1$ and that, due to the homogeneity of $F, F(x, y, d x / d s, d y / d s) d s=F(x, y, d x / d x(d x / d s)$, $d y / d x(d x / d s) d s=F(x, y, d x / d x, d y / d x)(d x / d s) d s=$ $F\left(x, y, 1, y^{\prime}\right) d x$, we obtain $F_{0}\left(x, y, y^{\prime}\right)=\mu\left[1+y^{\prime 2}+\right.$ $\left.\left(r^{2}-x^{2}-y^{2}\right)^{-1}\left(x+y y^{\prime}\right)^{2}\right]^{1 / 2}$, for all $(x, y) \in D$.

Then, the corresponding premetric (8) becomes

$$
d_{F}(\mathbf{a}, \mathbf{b})=\min _{\mathbf{x} \in \Omega} \int_{a}^{b} \mu\left(1+y^{\prime 2}+\frac{\left(x+y y^{\prime}\right)^{2}}{r^{2}-x^{2}-y^{2}}\right)^{1 / 2} d x
$$

The geodesic arcs of (18) on $D$ are the projections of half-great-circles of the half-sphere $f(x, y)=\left(r^{2}-\right.$ $\left.x^{2}-y^{2}\right)^{1 / 2}$ onto $D$. Thus, the distance $d_{F}(\mathbf{a}, \mathbf{b})$ from $\mathbf{a}=\left(a_{1}, a_{2}\right) \in D$ to $\mathbf{b}=\left(b_{1}, b_{2}\right) \in D$ is the product of $\mu$ and the Euclidean length of the geodesic arc connecting $\mathbf{a}$ and $\mathbf{b}$. That is to say, $d_{F}(\mathbf{a}, \mathbf{b})$ is the product of $\mu$ and the length of the half-great-circle segment going from $\left(a_{1}, a_{2},\left(r^{2}-a_{1}{ }^{2}-a_{2}^{2}\right)^{1 / 2}\right)$ to $\left(b_{1}\right.$, $\left.b_{2},\left(r^{2}-b_{1}{ }^{2}-b_{2}{ }^{2}\right)^{1 / 2}\right)$ on the surface of the halfsphere. Hence $d_{F}(\mathbf{a}, \mathbf{b})=\mu r \theta$, where $\theta=$ $\cos ^{-1}\left[\left(a_{1} b_{1}+a_{2} b_{2}+\left(r^{2}-a_{1}{ }^{2}-a_{2}{ }^{2}\right)^{1 / 2}\left(r^{2}-b_{1}{ }^{2}-\right.\right.\right.$ $\left.\left.b_{2}{ }^{2}\right)^{1 / 2}\right) / r^{2}$. Therefore, the premetric $d_{F}$ becomes

$$
\begin{aligned}
d_{F}(\mathbf{a}, \mathbf{b})= & \mu r \cos ^{-1}\left[\left(a_{1} b_{1}+a_{2} b_{2}+\left(r^{2}-a_{1}^{2}-a_{2}^{2}\right)^{1 / 2}\right.\right. \\
& \left.\left.\cdot\left(r^{2}-b_{1}^{2}-b_{2}^{2}\right)^{1 / 2}\right) / r^{2}\right] .
\end{aligned}
$$


This premetric is non-uniform and satisfies the conditions of symmetry, non-negativity and definiteness; i.e., $d_{F}$ in (19) is a non-uniform metric.

In order to take into account the action of gravity, we consider that the premetric given by (13), $d_{h}(\mathbf{a}, \mathbf{b})=$ $h(\mathbf{b}) \quad-h(\mathbf{a})$, represents the potential energy. Therefore, by Theorem 1, the sum $d_{F}+d_{h}$ is also a premetric $d$, and it is given by

$$
\begin{aligned}
d(\mathbf{a}, \mathbf{b}) & =h(\mathbf{b})-h(\mathbf{a})+\mu r \cos ^{-1}\left[\left(a_{1} b_{1}+a_{2} b_{2}\right.\right. \\
& \left.\left.+\left(r^{2}-a_{1}^{2}-a_{2}^{2}\right)^{1 / 2} \cdot\left(r^{2}-b_{1}^{2}-b_{2}^{2}\right)^{1 / 2}\right) / r^{2}\right]
\end{aligned}
$$

By Corollary of Theorem 6, the geodesic arcs of the premetric (20) and the geodesic arcs of the premetric (19) are the same.

If $h$ in (20) is a non-constant function, then the premetric given by (20) is asymmetric, non-uniform, violates the definiteness property, and it can take negative values.

\section{Conclusions}

We have given some theoretical basis and tools for modeling asymmetric, non-uniform and non-positive definite distance functions on $R^{n}$. Our approach is based on the fact that any distance function $d(\mathbf{a}, \mathbf{b})$ satisfying the triangle inequality can be obtained by solving a variational problem, where the integrand is a function $F_{0}(\mathbf{x}, \mathbf{v})$, called the fundamental function of $d$, depending on the position $\mathbf{x} \in R^{n}$ and on a direction $\mathbf{v} \in R^{n}$ attached at $\mathbf{x}$. The information contained in $d(\mathbf{a}$, b) is the same as that contained in its fundamental function $F: d$ gives "global information" because each value of it depends on the pair of points in $R^{n}$, and $F$ gives "local information" because each of its values depends on a point in $R^{n}$ and a direction that emanates from that point.

In general, the distance functions have been modeled through the distances between points of a sample of empirical data consisting of a heap of ordered pairs of points $(\mathbf{a}, \mathbf{b})$ on a given region. However, when we do not have any a priori information about the distance function that we want to model, it may be advantageous to model it through a fundamental function $F_{0}(\mathbf{x}, \mathbf{v})$ because it is less difficult to systematize local information corresponding to a heap of points $\mathbf{x}$ in the region and the directions $\mathbf{v}$ emanating from them than to find $a$ pattern for a heap of ordered pairs of points $(\mathbf{a}, \mathbf{b})$ in the region.

\section{References}

[1] Brimberg J., Walker J. H., Love R. F., Estimation of Travel Distances with the Weighted Lp norm: Some Empirical Results, Journal of Transport Geography, Vol. 15,2007 , pp. $62-72$

[2] Üster H., and Love R. F., Formulation of Confidence Intervals for Estimated Actual Distances, European Journal of Operational Research, Vol. 151, 2003, pp. 586601

[3] Berens W. and Körling F., Estimating Road Distances by Mathematical Functions, European Journal of Operational Research, Vol. 21, 1985, pp. 54-56.

[4] Brimberg J. and Love R. F, A New Distance Function for Modeling Travel Distances in a Transportation Network, Transportation Science, Vol. 26, No. 2, 1992, pp. $129-137$

[5] Brimberg J. and Love R. F., General Considerations on the Use of the Weighted Lp Norm as an Empirical Distance Measure, Transportation Science, Vol. 27, No. 4, 1993, pp. 341-349

[6] Love R. F and Morris J. G., Mathematical Models of Road Travel Distances, Management Sciences, Vol. 25, 1979, pp. 130-139

[7] Sánchez-Larios Hérica, Guillén-Burguete Servio, Modeling of Distance Functions on a Manifold, Nonlinear Analysis: Real World Applications, Vol.11, No. 4, 2010, pp. $3001-3007$

[8] A. Bóna, M. A. Slawiński, Raypaths as Parametric Curves in Anisotropic, Nonuniform Media: DifferentialGeometry Approach, Nonlinear Analysis TMA, Vol. 51, No. 6, 2002, pp. 983-994

[9] Kristály A., Moroşanu, Róth Á, Optimal Placement of a Deposit Between Markets: Riemann-Finsler Geometrical Approach, J Optim Theory Appl, Vol. 139, 2008, pp. 263276

[10] M. J. Hodgson, R. T. Wong, J. Honsaker, The pCentroid Problem on an Inclined Plane, Operations Research, Vol. 35, 1987, pp. 221-233 


\section{Authors' Biographies}

\section{Herica SANCHEZ-LARIOS}

Herica Sanchez-Larios received the master's and Ph. D. degrees in systems engineering (Operations Research) from the Universidad Nacional Autónoma de México (UNAM). She is a professor of the Graduate Program of Systems Engineering of the UNAM. Her research areas of interest are modeling of distance functions, facility location theory, and scheduling problems.

\section{Servio Tulio GUILLEN-BURGUETE}

Servio T. Guillen-Burguete received his master's degree in control and his engineering doctor's degree from the UNAM. His current research projects are related to decision theory, operation research and applied mathematics. He is a member of the National System of Researchers (SNI), the National Academy of Engineering (ANI), and the Mexican Association of Automatic Control (AMCA). 